\title{
Explosive Pulsed Power: An Enabling Technology
}

\author{
L.L. Altgilbers* \\ US Army Space and Missile Defense Command/Army Strategic Command \\ Huntsville, AL 35807, USA
}

\begin{abstract}
Explosive pulsed power consists of those devices that convert the chemical energy in explosives into electrical energy. In 2004, a series of programs were initiated to develop several types of very compact explosive pulsed power generators. Based on these recent efforts, we now have a better understanding of the weaknesses and strengths of these small generators. As a result, we can now build reliable generators that provide consistent output currents and voltages. In this paper, a brief introduction to these generators will be given along some of the most recent advances in our understanding of them. A description of an explosive driven high power microwave test bed being built at Texas Tech will be presented. This test bed can be used to test the effects of electromagnetic radiation on electronics. A brief description of some applications of explosive pulsed power will also be presented.
\end{abstract}

PACS numbers: 75.47.Lx, 75.47.Gk, 73.50.Fq

\section{Introduction}

Over the years, a number of explosive pulsed power devices were developed. In recent years, there have been significant improvements in explosive pulsed power (EPP) primarily due to the development of new materials [1-3] and to consistently funded experimental programs. Therefore, these explosive-driven systems are now being considered for a number of new applications including directed energy, powering special test equipment at remote test sites, rapid charging of capacitors, mine detection, propulsion, lightening and electromagnetic (EMP) simulators, electromagnetic launchers, mineral and oil exploration, and blasting operations at mines and quarries.

Of the 5 general classes of EPPs [4], only three will be considered in this paper and they include the following: magnetic flux compression generators (FCGs), ferroelectric generators (FEGs), and ferromagnetic generators (FMGs). These are the generators that appear to have practical applications at this time [4]. The FCG is a high energy source, the FEG is a high voltage source, and the FMG can be either a high voltage or a high current source depending on how it is built. The other FEGs and FMGs are relatively low energy sources.

\section{What is explosive pulsed power?}

Explosive pulsed power evolved out of the nuclear weapon programs in the United States, the United Kingdom, and the Soviet Union. These countries were looking for methods to solve several technical problems including driving fusion reactions without using a fission primer and driving detonator arrays and neutron sources.

Explosive pulsed power devices fall into one of two broad categories:

\footnotetext{
* e-mail: larry.altgilbers@smdc.army.mil
}

- Devices that convert the chemical energy of explosives into electrical energy by driving a conducting medium through a magnetic or an electric field. The basic process is to convert the chemical energy of the high explosives into electrical energy. This is accomplished by absorbing the kinetic energy of the detonation and transferring it to a moving conducting material. This moving conduction boundary distorts either an electric or magnetic field followed by a conversion into electrical energy. We will call these generators field interaction generators.

- Devices that use the shock waves generated by high explosives to induce a phase change in a material that stores energy in the form of electric or magnetic fields. We will call these generators phase transition generators.

\section{Generator description}

FCGs (see Fig. 1) use the chemical energy from high explosives to accelerate a metallic conductor, called the armature that traps and compresses a magnetic field initially created by a seed energy source such as a capacitor bank, battery, or another pulsed power generator. The accelerating armature compresses the seed magnetic field trapped within a conducting shell that is comprised of the armature, a stationary conductor called the stator, and end glide planes. When the armature makes electrical contact with the input glide plane, the initial magnetic flux from the seed source is trapped, and the seed circuit is disconnected from the generator. This process is called crowbarring. When the stator makes contact with the stator, a moving electrically conducting contact point is established, which closes the armature-stator circuit. If the FCG is a helical generator, the contact point propagates along the wire of the helical coil (stator) as the expanding conically shaped armature propagates along the axis of the generator. 


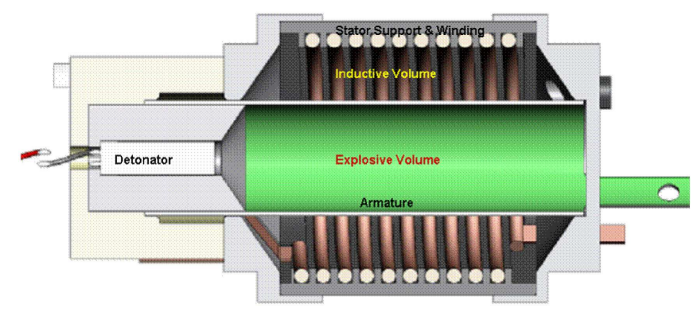

Fig. 1. Flux compression generator.

Compression of the trapped magnetic flux multiplies the initial seed current flowing in the stator. This amplified current is then delivered to a load either directly or through a power conditioning circuit. The energy density of an FCG, i.e. the ratio of the electrical energy delivered to the load and the FCG volume, is typically a few $\mathrm{J} / \mathrm{cc}$.

Note, however, that this number is critically dependent on the generator design and the load itself. In general, the smaller the load inductance, the higher the energy output. However, if the load inductance is too small, then the FCG cannot efficiently drive a load such as a narrow band microwave source or antenna.

FEGs use the chemical energy from high explosives to generate a shock wave. Ferroelectric materials store electrical energy when they are externally poled by an electric field. When a shock wave passes through the poled material, it causes a phase change in the crystalline structure. This phase change either depoles the material or causes the material to transition from one crystalline state to another and releases the stored electrical charge (or energy) via electrodes attached to the ferroelectric element to an external circuit. This released electric charge (or energy) is then delivered to a load via a power conditioning circuit. This type of generator can be used to deliver high voltages to high impedance loads and is suitable for the direct drive of radiating circuits.

FMGs use the chemical energy from high explosives to generate a shock wave to de-magnetize a permanent magnet. Ferromagnetic materials store energy in the form of a magnetic field when they are externally magnetized. When a shock wave passes through the ferromagnetic material, it destroys the magnet and the magnetic domains within the magnet. This changing magnetic field induces a current in a pickup coil around the magnet, which is then delivered to a load via a power conditioning circuit. This type of generator can be used to deliver large currents to low impedance loads and can generate higher voltages for moderate impedance loads.

The FCG is a field interaction type generator, while the FEG and FMG are phase transition type generators.

\section{Recent advances in EPP generators}

As noted earlier, the development of new types of materials and sustained experimental programs have led to significant improvements in our understanding of systems based on EPP and, in some cases, breakthroughs in improving their performance.

\subsection{Flux compression generators}

There are several different variants of small FCGs, but they all operate on the same basic principle of compressing a magnetic field in an enclosed conducting volume or magnetic field flux trap. They differ primarily in the shape of their conductors, which is limited by the types of explosive initiation systems that are available. In other words, practical initiation systems may not be possible for some geometric configurations.

\subsubsection{Armature studies}

While studying the propagation of the armature of an end fired helical FCG, Worsey et al. [5] observed the formation of a new type of fracture in expanding armatures and was able to answer questions about the impact of armature defects and voids in the explosives on generator operation. Based on his studies, he was able to explain why simultaneously initiated radially driven armatures are different from end fired axially propagating expanding armatures in conventional helical FCGs and, thus, how to deal with this difference to offset some of their more major problems.

The main areas that Baird focused his research on were the impact of the following on generator losses:

Expansion and fracturing of the armature. Examination of the high-speed photography of the expanding armatures revealed a previously unknown longitudinal cracking on the outer surface of the armatures. These cracks appeared in both types of metals, no matter what their annealed state. These longitudinal cracks began on the surface of the armature at the detonator end of the cylinder and always stopped their extension at identical distances along the cylinders. Since the armature is part of the generator's electric circuit and since the electric currents flow in a circumferential direction along its outer surface, it was thought that this might be one of the generator's loss mechanisms. The formation of cracks would introduce a loss of containment and result in magnetic flux losses. In summary, only detonation wave phenomenon, such as transmission, reflection, refraction, and trailing rarefactions, are capable of producing incipient fractures at the locations and times where the cracking began on the outer surface of the armatures. The longitudinal fractures are caused by shock waves, not the expansion due to the detonation. The expansion only opens the fractures once they are initiated.

Armature defects. Tests were conducted using copper and aluminum armatures with defects by using armatures that had been polished and those that had rough finishes. It was found that the surface finish had little or no effect on the armature's expanding surface.

Explosive packing and voids. Tests established that concerns about hand-packing were unfounded, as long as care was exercised to ensure that portions of explosive charge were knitted closely with previously loaded 
portions to prevent armature surface irregularities during expansion and that the only voids that appeared to effect armature expansion were those located at or near the explosive-armature interface.

\subsubsection{Shock wave sources}

Shock wave generators (SWGs) are very similar to flux compression generators, with the exception that magnetic field compression occurs within a solid material, such as aluminum powder, with its insulating oxide coating, or other suitable dielectric or semiconductor that becomes electrically conducting under the influence of a strong shock, rather than in a gas.

Some recent experimental advances are the following:

- While investigating shock induced changes in the electrical conductivity of aluminum powder for use in closing switches for ultracompact explosive generators such as FEGs, Hemmert found that the change in conductivity is independent of grain size, but may be influenced by the method used to pack the powder. In other words, the gain of the SWG is related to the porosity of the $\mathrm{Al}$ powder.

- Hemmert et al. [6] also found that high voltage hold-off tests showed a large difference in hold-off capabilities for different grain sizes, with the larger grains performing the worst. They concluded that the use of aluminum powder in compact explosive closing switches, based on metallization of the switch material, appeared possible for low voltage applications and that the use of nanopowders may achieve higher hold-off voltages.

\subsection{Ferroelectric generator}

Some of the recent advances in FEGs are the identification of:

New ferroelectric materials with higher energy storage densities and higher electric breakdown thresholds that significantly increase the output voltage of the FEG.

New potting materials that yield good electrical, mechanical, and shock properties.

Improved power conditioning techniques that yield optimal output voltages and provide better impedance matching with a variety of loads.

\subsection{Ferromagnetic generators}

The first paper describing explosive driven FMGs was published by Neilson [7] in 1957. Ferromagnetic generators may be classified as being either high current (kA) or high voltage $(\mathrm{kV})$ sources. Increasing the number of turns in the output coil of the FMG increases its output voltage. Thus, a single turn FEG is a high current source, while a multi-turn FMG is a high voltage source.

\section{Applications}

\subsection{Explosive-driven HPM test bed}

Texas Tech University is developing a compact, explosive-driven high power microwave (HPM) test bed. The major design constraints $[8,9]$ were that the system had to:
- Be completely self-contained; i.e., no external power source,

- Fit into a volume with a diameter no greater than $15 \mathrm{~cm}$ and a length no greater than $1.5 \mathrm{~m}$, and

- Radiate energy.

The primary objectives were to develop and optimize the various components of the system, study the issues associated with system integration, and train students to work with EPP. Of these three objectives the training of students is the most important. Explosive pulsed power is a multidisciplinary subject requiring training in high explosives, high voltage engineering, general electrical engineering, material science, vacuum engineering, and so on.

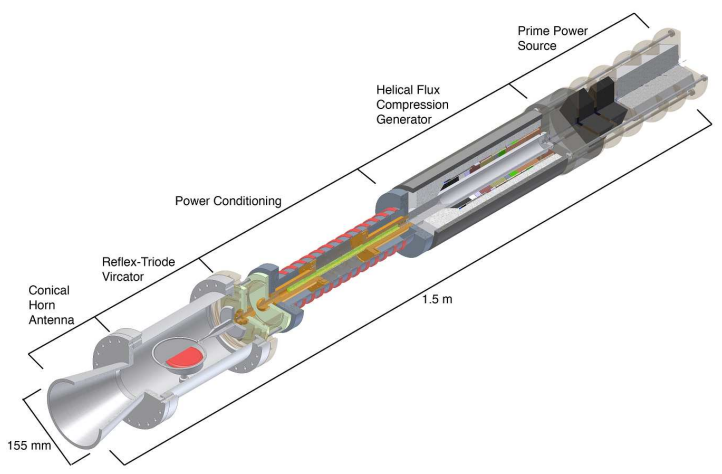

Fig. 2. Texas Tech explosive driven HPM test bed.

The major components of the test bed are shown in Fig. 2. As can be seen, it consists of a prime power or seed source for the flux compression generator, a helical FCG, a power conditioning module, a microwave source, and an antenna.

\subsection{Experimental observation of $R F$ radiation generated by an FEG driven antenna}

In 2005, the Naval Research Laboratory and Loki Inc. [10] conducted a series of tests in which they used an FEG to drive an antenna through a simple pulse forming network. They conducted three test shots using the same dipole antenna and pulse forming network and FEGs that had identical or similar physical configurations. A similar receiving dipole antenna was placed approximately $3 \mathrm{~m}$ from the transmitting antenna. The received wave forms were recorded along with the voltage pulse delivered by the FEG to the pulse forming network.

Using the peak voltages picked up by the receive antenna, the peak power density at the antennas was $1.64 \mathrm{~W} / \mathrm{cm}^{2}$ and the effective radiated power (ERP) at the source antenna was $2 \mathrm{MW}$, assuming a near unity gain of the receiving antenna. The FEG generated about 2.4 MW. 
A fast Fourier transform (FFT) revealed that the spectrum of the RF bursts was concentrated between 18 and $26 \mathrm{MHz}$, with largest signal at $21.4 \mathrm{MHz}$, which is in good agreement with the predicted value of $21.67 \mathrm{MHz}$.

\section{Conclusions}

Our improved understanding of the failure mechanisms observed in FCGs has provided us with clues on how to improve their performance. Researchers now understand why medium size generators work better than small FCGs and why completely new designs, such as the SWG, must be developed.

Improvements in ferroelectric materials and potting materials have allowed us to build FEGs with diameters as small as $40 \mathrm{~mm}$ that can consistently generate open circuit voltages in excess of $100 \mathrm{kV}$. These FEGs have been used for a number of applications including charging capacitor banks and vector inversion generators and driving antennas to produce radiated energy.

Ferromagnetic generators with diameters less than $50 \mathrm{~mm}$ have been successfully used to seed FCGs. These generators have proven to be reliable and capable of providing highly repeatable pulses. This enables us to build very compact completely autonomous EPP systems based on FCGs to drive a variety of payloads.

It has been demonstrated that FCGs can drive high power microwave sources and that FEGs can direct drive antennas to produce radiated signals.

Finally, Texas Tech has created a test bed that incorporates all the major components of a self-contained explosive driven HPM system. This will enable them and other researchers to test new components, address integrations issues, and train students in the use of high explosives, high voltage engineering, and HPM.

\section{References}

[1] S. Balevičius, V. Pyragas, N. Žurauskienè, L.L. Altgilbers, IEEE Trans. Appl. Supercond. 14, 112 (2004).

[2] S. Balevičius, N. Žurauskienè, V. Stankevič, P. Cimmperman, S. Keršulis, A. Cesnys, S. Tolvaišienè, L.L. Altgilbers, Appl. Phys. Lett. 90, 212503/1 (2007).

[3] S. Balevičius, A. Deksnys, V. Jasutis, A. Paulavičius, A. Poškus, N. Šichtorov, Thin Solid Films 112, 75 (1984).

[4] L.L. Altgilbers, M.D.J. Brown, I. Grishnaev, B.M. Nova, I.R. Smith, I. Tkach, Y. Tkach, Magnetocumulative Generators, Springer-Verlag, New York 2000.

[5] P. Worsey, J. Baird, J. Rasty, in: Explosively Driven Pulsed Power, Helical Magnetic Flux Compression Generators, Ed. A. Neuber, Springer Verlag,Berlin 2005, p. 53.

[6] D. Hemmert, S. Holt, J. Krile, in: 10th Annual Directed Energy Symp., Huntsville (AL, USA) 2007.

[7] F.W. Neilson, Sandia Technical Report SCTM-230B56(51), 1956.

[8] A. Young, M.Sc. Thesis, Texas Tech University, 2008.

[9] T.A. Holt, Ph.D. Thesis, Texas Tech University, 2008.

[10] M.S. Rader, C. Sullivan, T.D. Andreadis, Naval Research Laboratory Report NRL/FR/5745-05-10, 2005, p. 122. 